\title{
BOOTSTRAPPING REGULARITY OF THE ANOSOV SPLITTING
}

\author{
BORIS HASSELBLATT
}

(Communicated by Kenneth R. Meyer)

Abstract. Finite smoothness of the Anosov splitting implies $C^{\infty}$.

Definitions. Let $M$ be a compact Riemannian manifold. $f \in \operatorname{Diff}^{\infty}(M)$ is called Anosov with Anosov splitting $T M=E^{u} \oplus E^{s}$ if

$$
\begin{aligned}
& (\exists C, \epsilon>0), \\
& (\forall p \in M) \quad\left(\exists \mu_{1}<\mu_{2}<1-\epsilon<1+\epsilon<\nu_{2}<\nu_{1}\right) \\
& \left(\forall v \in E^{s}(p), u \in E^{u}(p), n \in \mathbb{N}\right), \\
& \left(\mu_{1}^{n} / C\right)\|v\| \leq\left\|D f^{n}(v)\right\| \leq C \mu_{2}^{n}\|v\| \text { and } \\
& \left(\nu_{1}^{-n} / C\right)\|u\| \leq\left\|D f^{-n}(u)\right\| \leq C \nu_{2}^{-n}\|u\| .
\end{aligned}
$$

$f$ is called $\alpha$-bunched if $\sup _{p \in M} \mu_{2} \nu_{2}^{-1}\left(\min \left(\mu_{1}, \nu_{1}^{-1}\right)\right)^{-\alpha}<1$.

Riemannian metrics with $C^{\infty}$-conjugate geodesic flows are called isodynamic.

Theorem. If $\sup _{p \in M} \nu_{1} \mu_{1}^{-1} \mu_{2}^{n}<1$ and $E^{u} \in C^{n}$ then $E^{u} \in C^{\infty}$.

The same holds for the weak unstable distribution of Anosov flows.

Katok obtained a similar result via nonstationary normal forms (oral communication). Since our technique is standard [HK, LMM], we only sketch the proof. We first list corollaries. Considering $f^{-1}$ yields

Corollary 1. If $\sup _{p \in M} \nu_{1} \mu_{1}^{-1} \nu_{2}^{-n}<1$ and $E^{s} \in C^{n}$ then $E^{s} \in C^{\infty}$.

Corollary 2. If $f$ preserves volume, $\operatorname{codim} E^{u}=1$, and $E^{u} \in C^{2}$, then $E^{u} \in$ $C^{\infty}$.

Remark. Ghys [G1] proves that a volume preserving Anosov flow on a compact manifold of dimension greater than three is a suspension if $\operatorname{codim} E^{u}=1$ and $E^{u} \in C^{2}$. Corollary 2 suggests that his result might be a rigidity statement.

Corollary 3. If $\operatorname{dim} M=2$ and $-\log \left\|D f^{k}{ }_{\left.\right|_{E^{u}(p)}}\right\| / \log \left\|D f^{k}{ }_{\Gamma_{E^{s}(p)}}\right\|<n-1-\delta$ for all $k \in \mathbb{N}, p \in M$, and $E^{u} \in C^{n}$, then $E^{u} \in C^{\infty}$.

Remark. Thus Theorem C of Ghys [G2] about the "generalized algebraicity" of flows with $C^{\infty}$ Anosov splitting holds with a $C^{3}$-assumption.

Received by the editors April 4, 1990.

1991 Mathematics Subject Classification. Primary 58F15, 58F17, 58F18; Secondary 53C12, $53 \mathrm{C} 20,53 \mathrm{C} 35$.

Key words and phrases. Differential geometry, dynamical systems, Anosov systems, hyperbolic systems, negative curvature, horospheric foliations. 
Corollary 4. If $n>2$ then $\frac{4}{n}$-bunched symplectic Anosov systems with $C^{n}$ Anosov splitting have $C^{\infty}$ Anosov splitting.

Proof. For symplectic Anosov systems $\mu_{i}^{-1}=\nu_{i}$.

Fact. [Kl] A $\gamma$-pinched Riemannian metric (where $\Lambda<$ sectional curvature $\leq \gamma \Lambda$ for some $\Lambda<0$ ) has $2 \sqrt{\gamma}$-bunched Anosov geodesic flow.

Corollary 5. Kanai's theorem $[\mathrm{K}]$ holds with a $C^{3}$ hypothesis.

Corollary 6. If $n>2$ then $\frac{4}{n^{2}}$-pinched Riemannian metrics with $C^{n}$ horospheric foliations are isodynamic to a locally symmetric metric.

Proof. By Corollary 4 this follows from the fact [BFL, F, FK, K] that metrics with $C^{\infty}$ horospheric foliations are isodynamic to a locally symmetric metric.

Proof. $E^{u}, E^{s}$ are tangent to foliations $W^{u}, W^{s}$ with smooth leaves. Around $p \in M$ introduce local $C^{\infty}$ coordinates $\psi_{p}: q \mapsto(x, y) \in \mathbb{R}^{j} \oplus \mathbb{R}^{l}$ (continuous in $p$ in the $C^{\infty}$-topology) so that $q \in W^{u}(p) \Leftrightarrow \psi_{p}(q)=(x, 0)$ and $q \in$ $W^{s}(p) \Leftrightarrow \psi_{p}(q)=(0, y)=: y_{0} \in\{0\} \oplus \mathbb{R}^{l}$ (for these coordinates and for flows refer to $[\mathrm{H}])$. Identify $E^{u}(q)$ via $D_{q} \psi_{p}$ with an affine subspace $E^{u}\left(y_{0}\right)$ of $\mathbb{R}^{j} \oplus \mathbb{R}^{l}$ containing $y_{0}$. Since $E^{u}(0)=\mathbb{R}^{j} \oplus\{0\}$ and by continuity, $E^{u}\left(y_{0}\right)$ is, after parallel translation, the graph of a linear map $E\left(y_{0}\right): \mathbb{R}^{j} \rightarrow \mathbb{R}^{l}$ or the image of $\left(\begin{array}{c}I \\ E\left(y_{0}\right)\end{array}\right): \mathbb{R}^{j} \rightarrow \mathbb{R}^{j} \oplus \mathbb{R}^{l}$, where $I$ is the identity of $\mathbb{R}^{j} . E^{u}(f q)$ is, via $\psi_{f p}$, the image of $\left(\begin{array}{c}I \\ E\left(y_{1}\right)\end{array}\right): \mathbb{R}^{j} \rightarrow \mathbb{R}^{j} \oplus \mathbb{R}^{l}$, which since $E^{u}(f q)=D_{q} f E^{u}(q)$ and $D_{q} f$ is (by invariance of $W^{s}(p)$ ) lower block triangular in local coordinates, coincides with that of

$$
D_{q} f\left(\begin{array}{c}
I \\
E\left(y_{0}\right)
\end{array}\right)=\left(\begin{array}{cc}
a_{y_{0}} & 0 \\
b_{y_{0}} & c_{y_{0}}
\end{array}\right)\left(\begin{array}{c}
I \\
E\left(y_{0}\right)
\end{array}\right)=\left(\begin{array}{c}
a_{y_{0}} \\
b_{y_{0}}+c_{y_{0}} E\left(y_{0}\right)
\end{array}\right)
$$

whence, by reparametrizing the preimage by $a_{y_{0}}^{-1}$,

$$
E\left(y_{1}\right)=c_{y_{0}} E\left(y_{0}\right) a_{y_{0}}^{-1}+b_{y_{0}} a_{y_{0}}^{-1} \text {. }
$$

Denote by $C_{s}^{k}$ the space of functions that are uniformly $C^{k}$ along stable leaves. We now show that if $k \geq n, E \in C_{s}^{k}$, and $\sup _{p \in M} \nu_{1} \mu_{1}^{-1} \mu_{2}^{n}<1$, then $E(\cdot) \in$ $C_{s}^{k+1}$. Differentiating (1) $k$ times yields

$$
\begin{gathered}
\left(E\left(y_{1}\right)\right)^{(k)}=\left(c_{y_{0}} E_{y_{0}} a_{y_{0}}^{-1}\right)^{(k)}+\left(b_{y_{0}} a_{y_{0}}^{-1}\right)^{(k)} \\
\text { or, with } \tilde{f}:=f_{\uparrow_{W^{s}(p)}}: W^{s}(p) \rightarrow W^{s}(p) \text { and } F_{p}^{i}:=\prod_{l=1}^{i}\left(D \tilde{f}\left(f^{l}(p)\right)\right)^{k}, \\
D_{s}^{k} E(f(p)) F_{p}^{1}=c_{p} D_{s}^{k} E(p) a_{p}^{-1}-\zeta(p)
\end{gathered}
$$

where $\zeta \in C^{1}$ since $\zeta$ is a polynomial in derivatives of $E$ up to order $k-1$ and those of $f$. Thus

$$
D_{s}^{k} E(p)=c_{p}^{-1} D_{s}^{k} E(f(p)) F_{p}^{1} a_{p}+c_{p}^{-1} \zeta_{p} a_{p}
$$

and recursively, with $A_{p}^{i}:=\prod_{l=0}^{i} a_{f^{i-l} p}$ and $C_{p}^{i}:=\prod_{l=0}^{i} c_{f^{\prime} p}^{-1}$,

$$
D_{s}^{k} E(p)=C_{p}^{m-1} D_{s}^{k} E\left(f^{m}(p)\right) F_{p}^{m} A_{p}^{m-1}+\sum_{i=0}^{m-1} C_{p}^{i} \zeta_{f^{i} p} F_{p}^{i-1} A_{p}^{i} .
$$


Since $\left\|C_{p}^{m-1}\right\| \leq C \mu_{1}^{-m},\left\|A_{p}^{m-1}\right\| \leq C \nu_{1}^{m}$ and $\left\|F_{p}^{m}\right\| \leq C \mu_{2}^{k m}$, we have

$$
D_{s}^{k} E(p)=\sum_{i=0}^{\infty} C_{p}^{i} \zeta_{f^{i} p} F_{p}^{i-1} A_{p}^{i} .
$$

$\left\|D_{z} C_{p}^{i-1}\right\| \leq C i \mu_{1}^{-i},\left\|D_{z} A_{p}^{i-1}\right\| \leq C i \nu_{1}^{i}$, and $\left\|D_{z} F_{p}^{i-1}\right\| \leq C i \mu_{2}^{k i}$ by the product rule, so derivatives of the terms of the above series have a convergent sum (uniformly in $p$ by exponential convergence) and $E \in C_{s}^{k+1}$. Inductively $E \in$ $C_{s}^{\infty}$. By a result of Journé [J] the fact that $W^{s}$ and $W^{u}$ are two continuous transverse foliations with uniformly smooth leaves implies that $C_{s}^{\infty} \cap C_{u}^{\infty}=$ $C^{\infty}$. Thus $E \in C^{\infty}$.

Proof of Corollary 2. If $\operatorname{dim} M=2$ see [HK], otherwise $\left\|C_{p}^{i} F_{p}^{i-1} A_{p}^{i}\right\|<C e^{-\epsilon i}$ and $D_{z}\left\|C_{p}^{i} F_{p}^{i-1} A_{p}^{i}\right\|<C i e^{-\epsilon i}$ for $k \geq 2, p \in M$, and the above argument applies.

Proof of Corollary 3. $\left\|C_{p}^{i} F_{p}^{i-1} A_{p}^{i}\right\|<C e^{-\epsilon i}$ and $D_{z}\left\|C_{p}^{i} F_{p}^{i-1} A_{p}^{i}\right\|<C i e^{-\epsilon i}$ for $k>n-1, p \in M$, and appropriate $\epsilon>0$.

\section{ACKNOWLEDGMENT}

I am indebted to the IHES for their hospitality and support while this paper was written, and to R. de la Llave for a helpful remark about [J].

\section{REFERENCES}

[BFL] Yves Benoist, Patrick Foulon, and François Labourie, Flots d'Anosov a distributions stable et instable differentiables, École Polytechnique preprint M949.0690, 1990.

[F] Renato Feres, Geodesic flows on Manifolds of Negative Curvature with Smooth Horospherical Foliations, Ergodic Theory Dynamical Systems 11 (1991), no. 4, 653-686.

[FK] Renato Feres and Anatoly B. Katok, Invariant tensor fields of dynamical systems with pinched Lyapunov exponents and rigidity of geodesic flows, Ergodic Theory and Dynamical Systems 9 (1989), 427-432; Anosov flows with smooth foliations and rigidity of geodesic flows in three-dimensional manifolds of negative curvature, Ergodic Theory and Dynamical Systems 10 (1990), 657-670.

[G1] Etienne Ghys, Codimension one Anosov flows and suspensions, Dynamical Systems Valparaiso 1986, (Rodrigo Bamón, Rafael Labarea, and Jacob Palis Jr., eds.), Lecture Notes in Mathematics vol. 1331, Springer-Verlag, Berlin, Heidelberg, and New York, 1988, pp. 59-72.

[G2] Etienne Ghys, Déformations de flots d'Anosov et de groupes Fuchsiens, Annales de l'Institut Fourier (1992) (to appear).

[H] Boris Hasselblatt, Regularity of the Anosov splitting and of horospheric foliations, preprint IHES/M/91/1, 1991.

[HK] Steven Hurder and Anatoly B. Katok, Differentiability, rigidity and Godbillon-Vey classes for Anosov flows, Publications Mathematiques de l'IHES 72 (1990), 5-61.

[J] Jean-Lin Journé, A regularity lemma for functions of several variables, Rev. Mat. Ibero. 4 (1988), 187-193.

[K] Masahiko Kanai, Geodesic flows of negatively curved manifolds with smooth stable and unstable foliations, Ergodic Theory and Dynamical Systems 82 (1988), 215-240.

[Kl] Wilhelm Klingenberg, Riemannian geometry, Studies in Math., de Gruyter, 1982, p. 276.

[LMM] Rafael de la Llave, J. M. Marco, and R. Moriyon, Canonical perturbation theory of Anosov systems and regularity results for the Livsic cohomology equation, Ann. of Math. 123 (1986), 537-611.

Department of Mathematics, Tufts University, Medford, Massachusetts 02155

E-mail address: BHasselb@Jade.Tufts.edu 